\title{
Movement Disorders Syndromes Associated With Lacunar Infarcts, Clinical Case Series
}

Chitsaz A., MD.

Professor of Neurology Isfahan University of M edical Sciences, Iran.

*Corresponding Author: Email: Chitsaz@med.mui.ac.ir

Lacunar infarcts are small ( $<15 \mathrm{~mm}$ diameter) subcortical infarcts that results from occlusion of a single penetrating artery. Lacunar infarcts are associated with clinical lacunar syndromes, one of lacunar syndromes is movement disorders syndrome such as chore, dystonia, hemibolismus and asterixis. Several types of movement disorders have been described with small, deep infarct, majority of these syndrome is caused by penetrating small- artery disease. The movement disorder may appear as the only sign of the infarct or may develop later, after an initial syndrome, different in character, has resolved.

Hemichorea - hemiballismus is the most common of movement disorder due to lacunar infarcts.

The lacunar size infarcts found in different parts of the striatum including in head of the caudate nucleus and adjacent corona radiate, the subthalamic and the thalamus. The onset is typically abrupt and is usually- unaccompanied by other complaints. The chorea usually involves the forearm, hand, and fingers. In some cases, chorea has been delayed by some weeks or months after the initial occurrence of hemiparesis.

Dystonia: Two types of dystonia have been described in cases of lacunes, action- induced and focal dystonia.

Case I- in a 63 year old woman with lacunar stroke, with abrupt onset of chorea, chorea involves the foream, hand, and fingers, it was accompanied by hemiparesis that faded within 3 months even though the chorea persisted unchanged.

Case II- In a 75 years old man chorea was delayed by some weeks after initial occurrence of hemiparesis due to lacunar infarct.

Case III- in a 81 years old woman lacunar infarct, whose putaminal lesions was documented by CT scan, suffer choreic movements of the distal parts of the arm and leg that interfered with normal activity and prevented easy walking for more than a weeks, the examination revealed normal strength, sensation and reflexes.

Case IV- A 69 years old man, with lacunar infarct in adjacent corona radiate with action - induced dystonia in the left hand. The finger of the affected hand become flexed in to the palm, leaving only the Thumb free, strength of hand was normal . Activity exacerbated the movements. The left hand and forearm showed involuntary movements that produced an unusual posture.

Key words: disorders syndromes

DOI: $10.7575 /$ aiac.abcmed.ca1.33

A I Published Date: February 2017

AC Peer-review is under responsibility of the 9th Iranian Stroke Congress.

Published by Australian International Academic Centre, Australia

This published work is open access under the CC BY license.

Available online at www.abcmed.aiac.org.au 